\title{
Real-World Data: Ranibizumab Treatment For Retinal Vein Occlusion In The OCEAN Study
}

This article was published in the following Dove Press journal: Clinical Ophthalmology

\author{
Josep Callizo (iD) \\ Focke Ziemssen $\mathbb{D}^{2}$ \\ Thomas Bertelmann ${ }^{3}$ \\ Nicolas Feltgen $\mathbb{D D}^{3}$ \\ Jessica Vögeler ${ }^{4}$ \\ Mirja Koch ${ }^{4}$ \\ Nicole Eter ${ }^{5}$ \\ Sandra Liakopoulos ${ }^{6}$ \\ Steffen Schmitz- \\ Valckenberg ${ }^{7}$ \\ Georg Spital ${ }^{8}$
}

'Department of Ophthalmology, GeorgAugust University, Goettingen, Germany; ${ }^{2}$ Center for Ophthalmology, Eberhard Karl University of Tuebingen, Tuebingen, Germany; ${ }^{3}$ Georg-August-University Goettingen, University Eye Hospital, Goettingen, Germany; ${ }^{4}$ Novartis Pharma $\mathrm{GmbH}$, Clinical Research, Nuremberg, Germany; ${ }^{5}$ Department of Ophthalmology, University of Muenster Medical Center, Muenster, Germany; ${ }^{6}$ Department of Ophthalmology, University Hospital of Cologne, Cologne, Germany; ${ }^{7}$ Department of Ophthalmology, University of Bonn, Bonn, Germany; ${ }^{8}$ Augenzentrum Am St. Franziskus Hospital, Muenster, Germany

Correspondence: Josep Callizo Department of Ophthalmology, GeorgAugust University, Robert-Koch-Str. 40, Goettingen 37075, Germany

Tel +495513966776

Fax+4955I3966787

Email josep.callizo@med.uni-goettingen.de
Purpose: The non-interventional OCEAN study (NCT02194803) evaluated frequency and monitoring of ranibizumab injections for retinal vein occlusion (RVO) in routine practice in Germany.

Methods: RVO patients (including branch and central RVO (BRVO/CRVO)) receiving ranibizumab were included. Best-corrected visual acuity (BCVA) testing, imaging and treatment were performed at the investigators' discretion and documented over 24 months.

Results: Overall, 744 RVO patients (27\% BRVO, 16\% CRVO, remaining unspecified RVO) were included. For $74 \%$ of patients, data were available for the 12 -month visit and for $56 \%$ for the 24-month visit. Mean baseline BCVA was 52.0 Early Treatment for Diabetic Retinopathy Study (ETDRS) letters (BRVO: 55.9, CRVO: 43.9). BCVA improved rapidly within the first 3 months, reaching 64.3 letters at 12 months and 64.7 at 24 months. CRVO patients showed less improvement than those with BRVO. Patients received a median of 4 (5) injections over 12 (24) months, with $100 \%$ of patients receiving injections at baseline, $70 \%$ at Month 1 and $81 \%$ at Month 2 . Overall, $40 \%$ of patients demonstrated a $\geq 15$ letter increase within the first 3 months ( $42 \%$ BRVO, 46\% CRVO). Patients with low initial BCVA ( $<50$ letters) showed greater improvement than patients with higher baseline BCVA. Due to considerable loss to follow-up, the number of injections and optical coherence tomography (OCT) examinations were not associated with the change in BCVA.

Conclusion: Patients with RVO in routine practice in Germany received fewer injections and fewer OCT examinations than in clinical trials. CRVO patients showed less and later improvement compared to BRVO patients.

Keywords: retinal vein occlusion, RVO, macular edema, ranibizumab, anti-VEGF, realworld

\section{Introduction}

Branch retinal vein occlusion (BRVO) and central retinal vein occlusion (CRVO) can lead to vision loss due to macular edema (ME) and ischemia. ${ }^{1,2}$ CRVO more frequently leads to severe vision loss compared to BRVO. ${ }^{3,4}$ For all forms of ME after RVO, anti-vascular endothelial growth factor (VEGF) treatment is currently the recommended therapy.

A number of studies have proven the beneficial effect of VEGF inhibition with ranibizumab (Lucentis ${ }^{\circledR}$, Novartis Pharma). ${ }^{3,5-12}$ According to treatment recommendations of the German ophthalmological societies, RVO treatment with antiVEGF injections should be initiated with at least three monthly injections, with further injections performed based on a pro re nata (PRN, as needed) or treat and extend $(\mathrm{T} \& \mathrm{E})$ regimen. 
The OCEAN study (Observation of treatment patterns with LuCEntis and real life ophthalmic monitoring, including optional OCT in Approved iNdications) was a prospective, multicenter, non-interventional study conducted to assess the outcomes of repeated intravitreal ranibizumab injections for neovascular age-related macular degeneration, diabetic macular edema, and ME caused by RVO in routine clinical practice in Germany. Here, we report results for the OCEAN patients with RVO. The main aim of the present analysis is to assess best-corrected visual acuity (BCVA), treatment frequency, and disease monitoring by optical coherence tomography (OCT), and thereby to assess the impact of treatment decisions in routine clinical practice. These results may help physicians to improve treatment strategies for RVO patients with ME in routine clinical practice.

\section{Materials And Methods}

From December 2011 to January 2015, patients were recruited prospectively to participate in the multicenter, non-interventional OCEAN study (NCT02194803) in Germany. The study was implemented in accordance with the Declaration of Helsinki. Ethics committee approval was obtained (Ethik-Kommission an der Medizinischen Fakultät der Eberhard-Karls-Universität und am Universitätsklinikum Tübingen; No: 618/2011BO2, 24. November 2011), and each patient provided written informed consent before enrolment. A total of 369 study sites (general ophthalmologists and tertiary care centers) across Germany participated. Design and patient demographics have been previously described. ${ }^{13}$ The full analysis set of the OCEAN study $(\mathrm{N}=5,641)$ included 744 patients with ME secondary to RVO. All patients were treated with intravitreal injections of ranibizumab $0.5 \mathrm{mg}$, with treatment and prescription performed at the physicians' discretion.

For the present analysis, data from all RVO patients were evaluated, including baseline disease characteristics, demographics, BCVA (which was repeatedly assessed by the physicians over the 2-year study period), along with treatment-related patterns such as the number of injections, clinic visits, and OCT examinations.

Previous treatment of the study eye with corticosteroids at any time or previous anti-VEGF treatment of the study eye within the 3 months before initiation of the study were exclusion criteria. Patients were categorized as treatmentnaïve, pre-treated and possibly pre-treated. Participants were treatment-naïve if their initial diagnosis of $\mathrm{ME}$ due to RVO occurred within the 3 months before entering the study and no treatment had been initiated. Patients with any documented anti-VEGF pre-treatment(s) were counted as pre-treated. All other patients were counted as possibly pretreated.

BCVA was documented by the physicians, and patients were stratified into groups by their initial BCVA $(<50,50$ to 65 , or $>65$ ETDRS letters). BCVA differences from baseline were explored using a paired sample $t$-tests and the impact of baseline BCVA on BCVA results was assessed by an analysis of variance (ANOVA) to evaluate possible trends. The time until study discontinuation, the time until treatment response (first improvement of $\geq 15$ letters from baseline), and the duration of treatment response were calculated by time-toevent analyses using Kaplan-Meier estimates.

To calculate variables involving time intervals (monthly study visits), an algorithm was implemented for sorting each patient's follow-up visits into a predefined visit schedule of 30 -day intervals ( \pm 15 days). Analyses were performed using an observed cases approach. In addition, to account for the effect of premature withdrawals and the different status of documentation, data for each patient at the last available visit were summarized in the form of a "last visit", ie using a last observation carried forward (LOCF) approach. Adverse events and concomitant diseases (anamnestic risk factors) were coded using version 19.1 of the Medical Dictionary for Regulatory Activities (MedDRA).

Analyses were purely descriptive and followed a predefined analysis plan.

\section{Results}

\section{Patient Population And Study Period}

A total of 744 patients with RVO (744 eyes) were included in the study. Participants had a mean age of 71 years, were $52 \%$ female, and $72 \%$ were treatment-naïve (Table 1). Of all RVO patients, 204 (27\%) were registered as BRVO and $121(16 \%)$ as CRVO patients. The remaining 419 cases $(56 \%)$ were documented by the treating physician solely as RVO, without further specification.

Anamnestic risk factors were explicitly documented for 514 patients $(69 \%)$ at baseline. The most frequently documented individual risk factors were hypertension (239 patients, 32\%) and diabetes mellitus (138 patients, 19\%). For $18 \%$ of the total RVO population, no risk factors were documented. These proportions were similar in the BRVO and CRVO subgroups.

The mean duration ( \pm standard deviation $[\mathrm{SD}]$ ) of the observational period after the first ranibizumab injection 
Table I Patient Demographics And Baseline Disease Characteristics Of OCEAN RVO Population (Full Analysis Set)

\begin{tabular}{|c|c|c|c|}
\hline & $\begin{array}{l}\text { Total RVO } \\
\text { Group } \\
\text { N=744 }\end{array}$ & $\begin{array}{l}\text { BRVO } \\
\text { Subgroup } \\
N=204\end{array}$ & $\begin{array}{l}\text { CRVO } \\
\text { Subgroup } \\
N=|2|\end{array}$ \\
\hline \multicolumn{4}{|l|}{ Gender [n (\%)] } \\
\hline Male & $353(47.45 \%)$ & $85(41.67 \%)$ & 57 (47.11\%) \\
\hline Female & $389(52.28 \%)$ & $119(58.33 \%)$ & $64(52.89 \%)$ \\
\hline Missing & $2(0.27 \%)$ & $0(0.00 \%)$ & $0(0.00 \%)$ \\
\hline \multicolumn{4}{|l|}{ Age [years] } \\
\hline $\mathrm{n}$ & 742 & 204 & 121 \\
\hline Mean (SD) & $71.0(10.9)$ & $71.2(10.0)$ & $70.3(11.5)$ \\
\hline \multicolumn{4}{|l|}{$\begin{array}{l}\text { Age, categorized } \\
\text { [n (\%)] }\end{array}$} \\
\hline$<65$ years & 199 (26.75\%) & $53(25.98 \%)$ & $33(27.27 \%)$ \\
\hline 65 to $<70$ years & $92(12.37 \%)$ & $26(12.75 \%)$ & $15(12.40 \%)$ \\
\hline 70 to $<75$ years & $151(20.30 \%)$ & 47 (23.04\%) & $23(19.01 \%)$ \\
\hline 75 to $<80$ years & $160(21.51 \%)$ & $43(21.08 \%)$ & $29(23.97 \%)$ \\
\hline 80 to $<85$ years & 85 (1 $1.42 \%)$ & $24(11.76 \%)$ & $14(11.57 \%)$ \\
\hline$\geq 85$ years & 55 (7.39\%) & II (5.39\%) & 7 (5.79\%) \\
\hline Missing & $2(0.27 \%)$ & $0(0.00 \%)$ & $0(0.00 \%)$ \\
\hline \multicolumn{4}{|l|}{ BMI $\left[\mathrm{kg} / \mathrm{m}^{2}\right]$} \\
\hline $\mathrm{n}$ & 692 & 196 & 119 \\
\hline Mean (SD) & $27.1(4.3)$ & $27.1(4.3)$ & $26.8(4.4)$ \\
\hline \multicolumn{4}{|l|}{ Type of RVO } \\
\hline BRVO & $204(27.42 \%)$ & - & - \\
\hline CRVO & $121(16.26 \%)$ & - & - \\
\hline Not specified & 419 (56.32\%) & - & - \\
\hline \multicolumn{4}{|l|}{$\begin{array}{l}\text { Pre-treatment } \\
\text { status }\end{array}$} \\
\hline Treatment-naïve & $536(72.04 \%)$ & 148 (72.55\%) & 87 (71.90\%) \\
\hline $\begin{array}{l}\text { Pre-treated with } \\
\text { anti-VEGF }\end{array}$ & $73(9.81 \%)$ & $19(9.31 \%)$ & $9(7.44 \%)$ \\
\hline Possibly pre-treated & $135(18.15 \%)$ & 37 (18.14\%) & $25(20.66 \%)$ \\
\hline \multicolumn{4}{|l|}{$\begin{array}{l}\text { Laser pre- } \\
\text { treatment prior to } \\
\text { first ranibizumab } \\
\text { treatment }\end{array}$} \\
\hline \multicolumn{4}{|l|}{$\begin{array}{l}\text { Time from diagnosis } \\
\text { of macular edema } \\
\text { due to RVO to first } \\
\text { injection in OCEAN } \\
\text { study [days] }\end{array}$} \\
\hline $\mathrm{n}$ & 727 & 201 & 118 \\
\hline Mean (SD) & $182.2(474.5)$ & $192.3(498.7)$ & $115.2(197.8)$ \\
\hline Median & 47 & 50 & 44 \\
\hline Min - Max & I - 5,386 & $7-3,87 \mid$ & $3-1,150$ \\
\hline
\end{tabular}

(Continued)
Table I (Continued).

\begin{tabular}{|c|c|c|c|}
\hline & $\begin{array}{l}\text { Total RVO } \\
\text { Group } \\
\mathbf{N = 7 4 4}\end{array}$ & $\begin{array}{l}\text { BRVO } \\
\text { Subgroup } \\
\mathrm{N}=204\end{array}$ & $\begin{array}{l}\text { CRVO } \\
\text { Subgroup } \\
\mathrm{N}=|2|\end{array}$ \\
\hline $\begin{array}{l}\text { BCVA of study eye } \\
\text { at baseline } \\
n \\
\text { Mean (SD) ETDRS } \\
\text { letters } \\
\text { Mean (SD) logMAR }\end{array}$ & $\begin{array}{l}736 \\
52.0(22.7) \\
0.659(0.454)\end{array}$ & $\begin{array}{l}203 \\
55.9(20.9) \\
0.583(0.4 \mid 8)\end{array}$ & $\begin{array}{l}120 \\
43.9(24.8) \\
0.823(0.496)\end{array}$ \\
\hline $\begin{array}{l}\text { BCVA of study eye } \\
\text { at baseline, } \\
\text { categorized [n (\%)] } \\
n \\
<50 \text { letters } \\
50 \text { to } 65 \text { letters } \\
>65 \text { letters } \\
\text { Missing }\end{array}$ & $\begin{array}{l}744 \\
(100.00 \%) \\
225(30.24 \%) \\
222(29.84 \%) \\
289(38.84 \%) \\
8(1.08 \%)\end{array}$ & $\begin{array}{l}204 \\
(100.00 \%) \\
43(21.08 \%) \\
63(30.88 \%) \\
97(47.55 \%) \\
I(0.49 \%)\end{array}$ & $\begin{array}{l}|2| \\
(100.00 \%) \\
56(46.28 \%) \\
32(26.45 \%) \\
32(26.45 \%) \\
\mid(0.83 \%)\end{array}$ \\
\hline $\begin{array}{l}\text { OCT performed at } \\
\text { baseline } \\
\text { Yes } \\
\text { No } \\
\text { Missing }\end{array}$ & $\begin{array}{l}558(75.00 \%) \\
184(24.73 \%) \\
2(0.27 \%)\end{array}$ & $\begin{array}{l}|6|(78.92 \%) \\
43(21.08 \%) \\
0(0.00 \%)\end{array}$ & $\begin{array}{l}86(71.07 \%) \\
35(28.93 \%) \\
0(0.00 \%)\end{array}$ \\
\hline $\begin{array}{l}\text { FA performed at } \\
\text { baseline } \\
\text { Yes } \\
\text { No } \\
\text { Missing }\end{array}$ & $\begin{array}{l}525(70.56 \%) \\
215(28.90 \%) \\
4(0.54 \%)\end{array}$ & $\begin{array}{l}\mid 46(71.57 \%) \\
58(28.43 \%) \\
0(0.00 \%)\end{array}$ & $\begin{array}{l}87(71.90 \%) \\
34(28.10 \%) \\
0(0.00 \%)\end{array}$ \\
\hline
\end{tabular}

Note: ${ }^{\mathrm{a}} \mathrm{A}$ total of 419 cases were documented by the treating physician solely as RVO, not defining whether a branch or the central vein was occluded.

Abbreviations: CVA, best-corrected visual acuity; BMI, body mass index; BRVO, branch retinal vein occlusion; CRVO, central retinal vein occlusion; ETDRS, Early Treatment for Diabetic Retinopathy Study; FA, fluorescein angiography; logMAR, logarithm of the minimum angle of resolution; Max, maximum; Min, minimum; $\mathrm{OCT}$, optical coherence tomography; RVO, retinal vein occlusion; SD, standard deviation; VEGF, vascular endothelial growth factor.

was $503.5 \pm 251.2$ days $(\mathrm{n}=662)$, ie approximately 17 months. For 550 patients (74\%), study data were available up to the 12-month visit and for 416 patients (56\%) up to the 24-month visit. The rate of premature discontinuation was relatively constant throughout the study (Figure 1C).

\section{Clinic Visits, BCVA Assessments, And OCT Examinations}

All 744 RVO patients had a recorded baseline visit, while the number of patients with documented data for the respective monthly visits decreased over the course of the study, as 

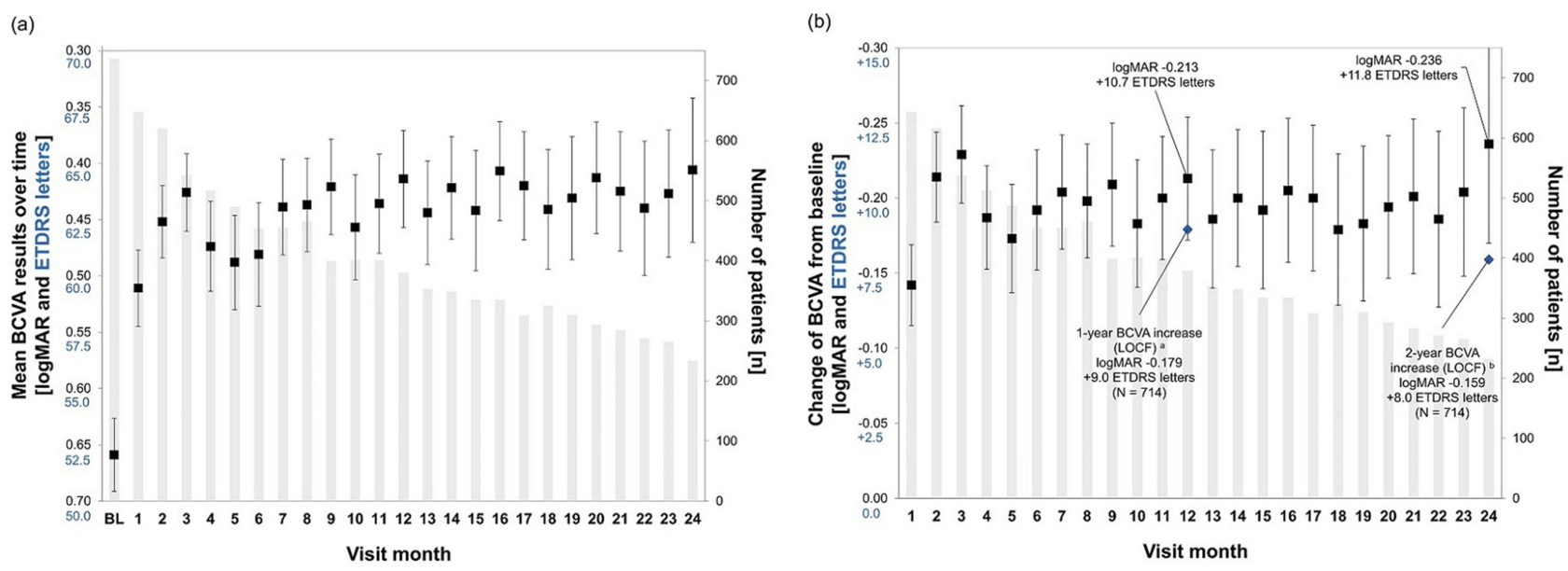

(c)

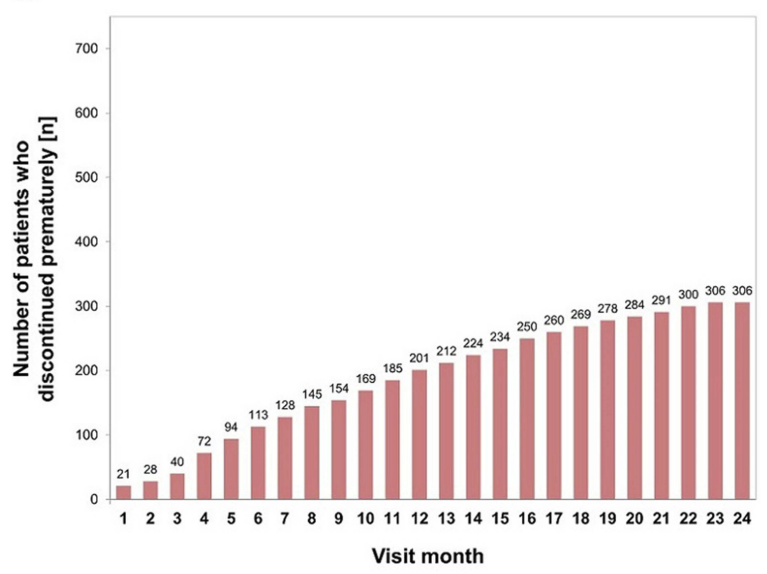

Figure I Visual acuity over the course of 24 months, overall RVO population. (A) Development of mean BCVA over time; (B) Mean difference in BCVA from baseline to study visits; (C) Number of patients who discontinued prematurely over the course of 24 months.

Notes: 'Last documented BCVA result in first year of study (LOCF). 'Last documented BCVA result in entire study period (LOCF). Boxes and error bars: mean values and 95\% confidence intervals; grey bars: number of patients per visit; red bars: numbers of patients who discontinued.

Abbreviations: BCVA, best-corrected visual acuity; BL, baseline; ETDRS, Early Treatment for Diabetic Retinopathy Study; LOCF, last observation carried forward; logMAR, logarithm of the minimum angle of resolution; RVO, retinal vein occlusion.

expected for an observational study. Over the course of the 2-year study, patients underwent a median of 15 BCVA examinations (range: 1-25; mean: 14.4), with 10 (range $0-13$; mean: 8.8 ) of these examinations performed during the first year (including baseline). A median of 2 (range 0-19; mean: 3.3) follow-up BCVA examinations were performed within 4 weeks after injection, which may be interpreted as safety check-ups, a common practice in Germany. A further 2 (range 0-20; mean: 3.0) BCVA examinations were performed 4 to 8 weeks after injection, whereas a median of 5 examinations (range 0-23; mean: 7.0) were performed later than 8 weeks. These monitoring visits after 4 or 8 weeks were presumably focused on the assessment of the need for re-injection.

In addition, patients underwent a median of 5 OCT examinations (range 0-25; mean: 6.6) during the 2-year study, with 3 (range $0-13$; mean 4.1) of these performed during the first year (including baseline). Among patients who had at least one OCT during the entire study (655 patients), the median number of OCTs was slightly higher over 2 years (6 OCTs, range 1-25; mean: 7.5). Of the follow-up OCTs, a median of 0 OCTs (range $0-14$; mean: 0.8 ) were performed within the first 4 weeks after injection, 1 OCT (range 0-20; mean: 1.9) was performed 4 to 8 weeks after, and 1 OCT (range 0-21; mean: 3.1) was performed later than 8 weeks. Mean central retinal thickness at baseline was $470.2 \pm 182.7$, and was higher in the CRVO subgroup (562.5 \pm 248.7$)$ than the BRVO subgroup (431.2 \pm 137.0$)$.

\section{Number Of Injections}

Overall, RVO patients received a median of 5 injections (range 1-22; mean: 5.99) during the 2-year observational 
period. This number of injections was comparable in the BRVO (5 injections [range 1-20, mean: 6.52]) and CRVO (5 injections [range 1-19; mean: 6.64]) subgroups.

The frequency of treatment decreased during the study period. During the first year, patients received a median of 4 injections (range 1-13; mean: 4.67) (BRVO subgroup: 4 injections [range 1-11; mean: 4.90]; CRVO subgroup: 5 injections [range 1-13; mean: 5.11]), with 3 injections (range 1-7; mean: 3.54) during the first 6 months of the study (BRVO subgroup: 3 injections [range 1-7; mean: 3.58]; CRVO subgroup: 3 injections [range 1-7; mean: 3.78]) and 0 injections (range 0-7; mean: 1.13) during the second 6 months (BRVO subgroup: 0 injections [range 0-5; mean: 1.32]; CRVO subgroup: 1 injection [range 0-7; mean: 1.33]). In the second year, the number of injections per 6 months decreased in all groups.

When assessing the ranibizumab "upload treatment phase", ie the first three months of the study, all 744 patients received an injection at baseline and the majority also received injections at Month $1(70 \%)$ and at Month 2 $(81 \%)$. Thereafter, the proportion of patients who received an injection decreased to around $30 \%$ or less at each month. Similar proportions were seen in the BRVO and CRVO subgroups.

\section{Visual Acuity (VA)}

The mean baseline BCVA in the overall RVO population was 52.0 22.7 ETDRS letters (Snellen fraction: $~ 6 / 27$ ), for the BRVO subgroup $55.9 \pm 20.9$ letters (Snellen fraction: $\sim 6 / 23$ ) and CRVO subgroup $43.9 \pm 24.8$ letters (Snellen fraction: $\sim 6 / 40$ ) (Table 1).

The distribution of initial BCVA differed slightly between the groups: in the overall RVO group and BRVO subgroup patients most frequently had an initial BCVA of $>65$ letters ( $39 \%$ and $48 \%$, respectively), while for the CRVO group $<50$ letters was most frequently reported (46\%) (Table 1).

The observed improvements in BCVA over the course of the study are provided in Table 2 and Figure 1A and B (LOCF data presented additionally). In the overall RVO population, a rapid improvement of BCVA was observed within the first 3 months of the study, with the mean BCVA reaching 63.7 ETDRS letters (Snellen fraction: 6/16). After that, the mean BCVA worsened slightly before increasing to 64.3 letters (Snellen fraction: 6/16) at Month 12 and 64.7 letters (Snellen fraction: 6/15) at Month 24. In the BRVO subgroup, similar improvements were observed by the end of the first year, followed by a slight deterioration in the second year of the study (Table 2 and Figure 2A and B). In contrast, the CRVO subgroup showed notably less improvement from baseline to the end of the first year, but then showed an increase during the second year, reaching a change from baseline ( +9 letters) comparable to the BRVO subgroup (+11 letters) by the end of the study. However, the absolute mean BCVA in the CRVO subgroup remained lower than in the BRVO subgroup (Table 2 and Figure 2A).

Table 2 Best-Corrected Visual Acuity Over The Course Of The OCEAN Study (Full Analysis Set)

\begin{tabular}{|c|c|c|c|c|c|c|}
\hline \multirow[t]{2}{*}{ BCVA } & \multicolumn{2}{|c|}{$\begin{array}{l}\text { Total RVO Group } \\
\mathrm{N}=744\end{array}$} & \multicolumn{2}{|c|}{$\begin{array}{l}\text { BRVO Subgroup } \\
N=204\end{array}$} & \multicolumn{2}{|c|}{$\begin{array}{l}\text { CRVO Subgroup } \\
N=|2|\end{array}$} \\
\hline & $\mathbf{n}$ & Mean (SD) ETDRS letters & $\mathbf{n}$ & Mean (SD) ETDRS letters & $\mathbf{n}$ & Mean (SD) ETDRS letters \\
\hline \multicolumn{7}{|l|}{ Baseline } \\
\hline BCVA & 736 & $52.0(22.7)$ & 203 & $55.9(20.9)$ & 120 & $43.9(24.8)$ \\
\hline \multicolumn{7}{|l|}{ Month 3} \\
\hline BCVA & 543 & $63.7(20.5)$ & 164 & $67.6(17.8)$ & 94 & $54.3(24.6)$ \\
\hline Change from baseline & 538 & $+11.5(19.4)$ & 163 & $+10.3(19.6)$ & 93 & $+13.2(22.0)$ \\
\hline \multicolumn{7}{|l|}{ Month I2 } \\
\hline BCVA & 380 & $64.3(21.5)$ & 139 & $69.1(14.6)$ & 71 & $52.1(28.7)$ \\
\hline Change from baseline & 379 & $+10.7(20.3)$ & 139 & $+13.1(19.0)$ & 71 & $+4.1(24.7)$ \\
\hline \multicolumn{7}{|l|}{ Month 24} \\
\hline BCVA & 234 & $64.7(24.9)$ & 93 & $65.6(23.8)$ & 47 & $57.2(33.9)$ \\
\hline Change from baseline & 232 & $+11.8(25.5)$ & 92 & $+11.4(26.2)$ & 47 & $+9.2(30.0)$ \\
\hline
\end{tabular}

Abbreviations: BCVA, best-corrected visual acuity; BRVO, branch retinal vein occlusion; CRVO, central retinal vein occlusion; ETDRS, Early Treatment for Diabetic Retinopathy Study; logMAR, logarithm of the minimum angle of resolution; RVO, retinal vein occlusion; SD, standard deviation. 
(a)

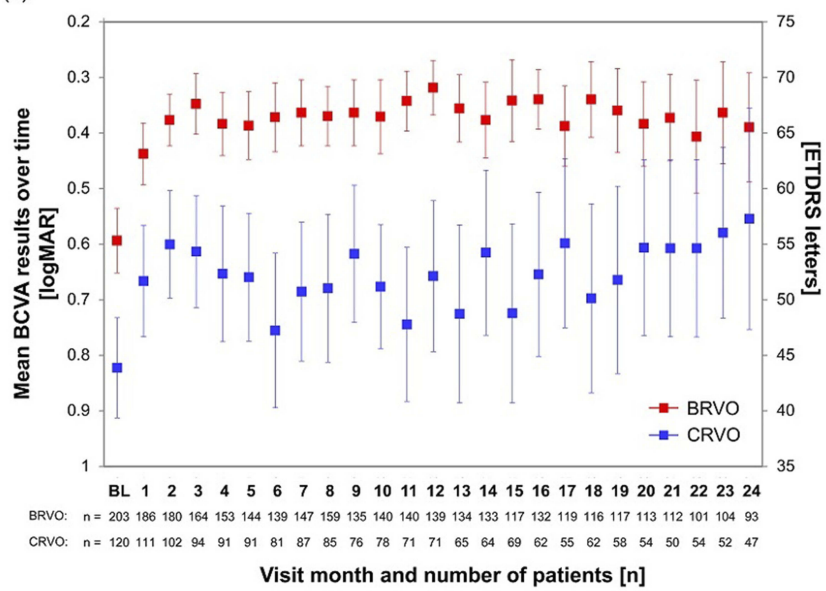

(b)

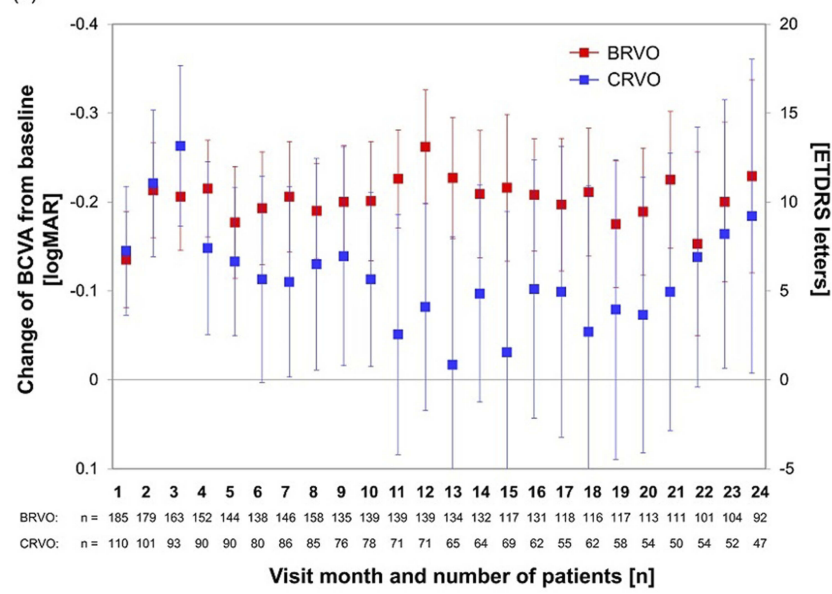

Figure 2 Visual acuity over the course of 24 months in BRVO and CRVO subgroups. (A) Development of mean BCVA over time; (B) Mean difference in BCVA from baseline to study visits.

Note: Boxes and error bars: mean values and $95 \%$ confidence intervals.

Abbreviations: BCVA, best-corrected visual acuity; BL, baseline; BRVO, branch retinal vein occlusion; CRVO, central retinal vein occlusion; ETDRS, Early Treatment for Diabetic Retinopathy Study; logMAR, logarithm of the minimum angle of resolution.

In the overall RVO population, $35 \%$ of the patients showed a BCVA improvement of $\geq 15$ letters, $54 \%$ showed a change between $>-15$ letters and $<15$ letters, and $8 \%$ showed a deterioration of $\geq 15$ letters at the end of the first year of the study (calculated using the LOCF approach). After the entire 2-year study period, the results were similar, with $35 \%$ of patients showing an improvement of $\geq 15$ letters and $10 \%$ showing a deterioration of $\geq 15$ letters.

BCVA results were in a similar range for the BRVO and CRVO subgroups, with slightly better results for BRVO. In the BRVO subgroup slightly more patients improved by $\geq 15$ letters (Month 12: 40\%, Month 24: 39\%) compared to CRVO (Month 12: 35\%, Month 24: $36 \%$ ), whereas in the CRVO subgroup more patients deteriorated by $\geq 15$ letters (Month 12: 14\%, Month 24: 17\%) compared to BRVO (Month 12: 5\%, Month 24: 8\%).

Additionally, the BCVA improvements were analyzed stratified by the patients' baseline BCVA (supporting information: Figure S1). Patients with low initial BCVA ( $<50$ letters) showed greater relative improvement compared to baseline (+20.9 letters at Month 12, +25.8 letters at Month 24) than patients with a baseline BCVA of 50-65 letters $(+11.6$ letters at Month 12, +13.2 letters at Month 24) or patients with a baseline BCVA of $>65$ letters ( +2.4 letters at Month $12,+0.2$ letters at Month 24).

Nevertheless, BCVA after 1 and 2 years of observation remained better in patients with higher baseline BCVA. Patients with a baseline BCVA $<50$ letters reached a mean
BCVA of 48.9 letters at Month 12 and 51.9 letters at Month 24 , while patients with a baseline BCVA of 50-65 letters reached 67.1 letters (Month 12) and 68.5 letters (Month 24), and patients with a baseline BCVA of $>65$ letters reached 73.0 letters (Month 12) and 71.2 letters (Month 24).

These differences in improvements from baseline per group were also seen in the BRVO subgroup, where the improvements in the lowest baseline BCVA group $(<50$ letters) were even more pronounced $(+31.8$ letters at Month 12, +31.7 letters at Month 24) than in the overall population. In contrast, the CRVO subgroup, which started the study with a lower mean BCVA with almost half of patients in the $<50$ letters baseline BCVA group (Table 1), showed much smaller improvements. However, the largest relative improvements in CRVO were also seen in patients with a baseline BCVA of $<50$ letters $(+10.8$ letters at Month 12, +15.4 letters at Month 24), whereas patients with a baseline BCVA of $>65$ letters even showed a deterioration of -3.9 letters at Month 12 and an increase of only +0.3 letters at Month 24 .

The BCVA differences from baseline were additionally analyzed by $t$-tests for the overall RVO population. The improvements in BCVA from baseline to Month 12 and Month 24 were statistically significant in patients with a baseline BCVA of $<50$ letters and of 50 to 65 letters ( $\mathrm{p}<0.0001$ for both groups and time points). In patients with a baseline BCVA of $>65$ letters, the improvements from baseline were significant at Month $12(\mathrm{p}=0.0181)$, but not at Month $24(\mathrm{p}=0.9260)$. 
An ANOVA was performed to analyze the differences between the baseline BCVA groups in more detail. At Month 12 and Month 24, the baseline BCVA $(<50,50$ to 65 , or $>65$ letters) had a statistically significant impact on the difference from baseline $(\mathrm{p}<0.0001)$. Statistically significant $p$-values $(p<0.05)$ were also found for all pairwise comparisons of the three groups at both time points.

In the overall population, BCVA results were further analyzed by the patients' pre-treatment status, showing higher improvements in treatment-naïve patients compared to pre-treated patients throughout the observational period. However, the mean BCVA was higher in pre-treated patients compared to treatment-naïve patients at each visit (supporting information: Figure S2). The mean baseline BCVA was $60.5 \pm 16.9$ ETDRS letters for pre-treated patients $(\mathrm{n}=73)$ and 50.6 \pm 23.2 ETDRS letters for treatment-naïve patients $(n=529)$. This difference between the two groups had decreased by Month 12 (pre-treated: 68.3 \pm 12.7 ETDRS letters [n=39], treatment-naïve: $64.5 \pm 21.9$ ETDRS letters [ $\mathrm{n}=276]$ ) and by Month 24 (pre-treated: $70.3 \pm 14.8$ ETDRS letters [n=22], treatment-naïve: 64.9 \pm 24.2 ETDRS letters [ $n=164]$ ). It should be taken into account that the pre-treated subgroup was notably smaller than the treatment-naïve subgroup. The 135 possibly pretreated patients (see Table 1) were not included in this analysis.

The BCVA improvements were also analyzed by number of OCT examinations. Patients who received more than 4 OCTs during the study tended to show minimally better response (in terms of gain of $\geq 5, \geq 10$, and $\geq 15$ letters) over the entire course of the study than patients who received 4 OCTs or fewer. However, further analyses of the impact of the number of OCTs on BCVA results, eg using different stratifications of patients by their number of OCTs, did not corroborate these findings. Thus, these results must be interpreted with caution.

The assessment of the BCVA results over time grouped by the number of injections received during the entire study did not reveal any consistent impact of treatment frequency on VA outcomes.

\section{Time-To-Event Analyses}

The rate of premature discontinuation was relatively constant throughout the study. The probability of an RVO patient remaining in the study up to Month 12 and Month 24 was approximately $73 \%$ and $59 \%$, respectively (Figure 3A). Results were similar in the CRVO subgroup. However, for the BRVO subgroup, the discontinuation rate was lower than for the overall RVO population (Figure 3B). Time to response and duration of response were additionally analyzed: approximately $40 \%$ of RVO participants had $a \geq 15$ letter response within the first 3 months of the study (Figure 4A). In participants who showed a $\geq 15$ letter response, the probability of a $\geq 15$ letter response lasting $\geq 3$ months was 59\% (Figure 5A). Time to response and duration of response did not notably differ between the BRVO and CRVO subgroups (Figures 4B and 5B).

\section{Adverse Events}

Adverse events were documented for $26 \%$ of all 764 RVO patients in the safety analysis set. The most frequent adverse event was intraocular pressure increased (41 patients) (a)

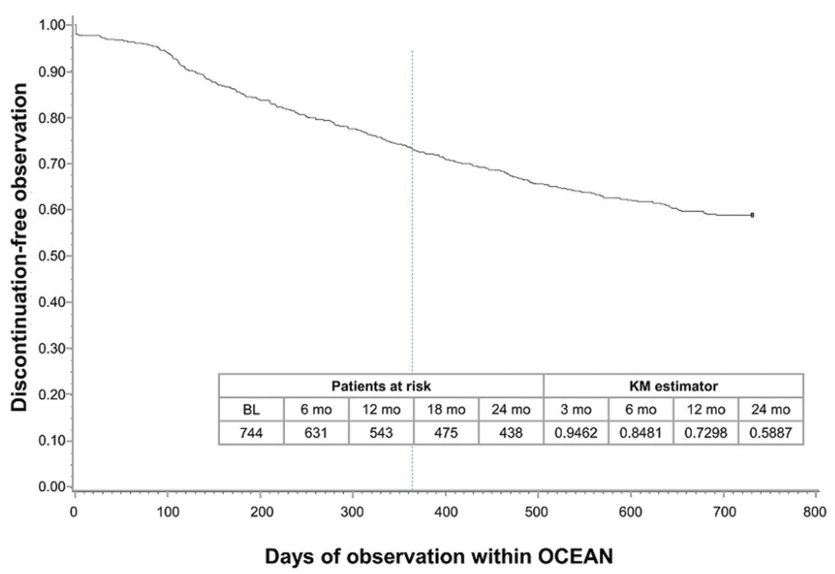

(b)

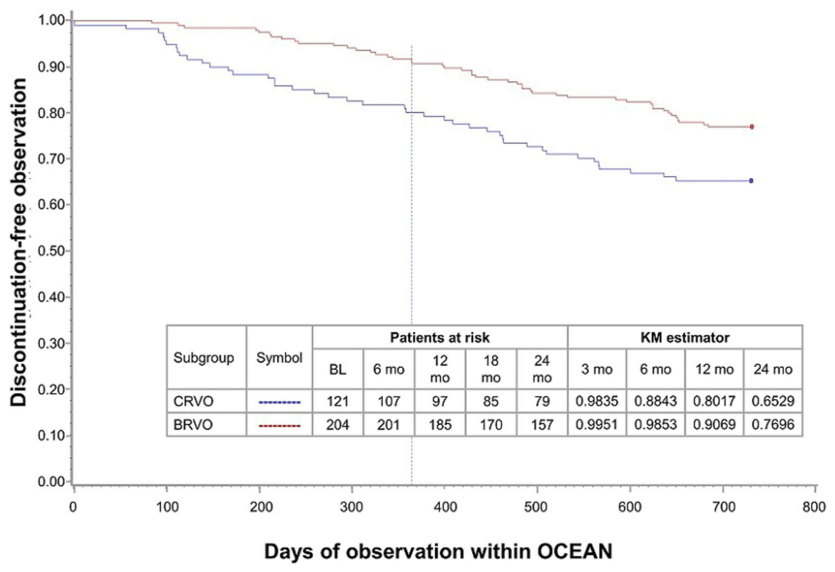

Figure 3 Time to discontinuation. (A) In all patients and (B) In BRVO and CRVO subgroups.

Notes: If the time of discontinuation was not documented, it was set to day I. Estimators are based on the Kaplan-Meier product-limit methodology.

Abbreviations: BL, baseline; BRVO, branch retinal vein occlusion; CRVO, central retinal vein occlusion; KM, Kaplan-Meier; mo, months. 
(a)

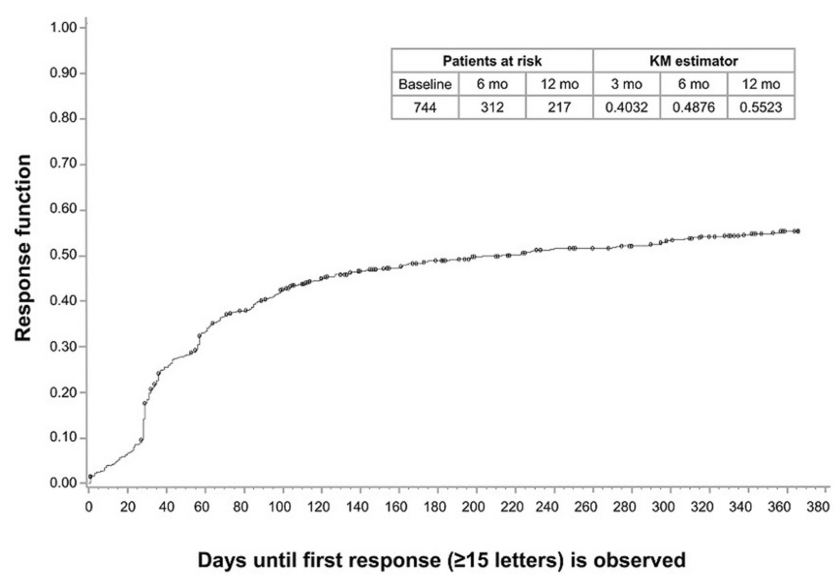

(b)

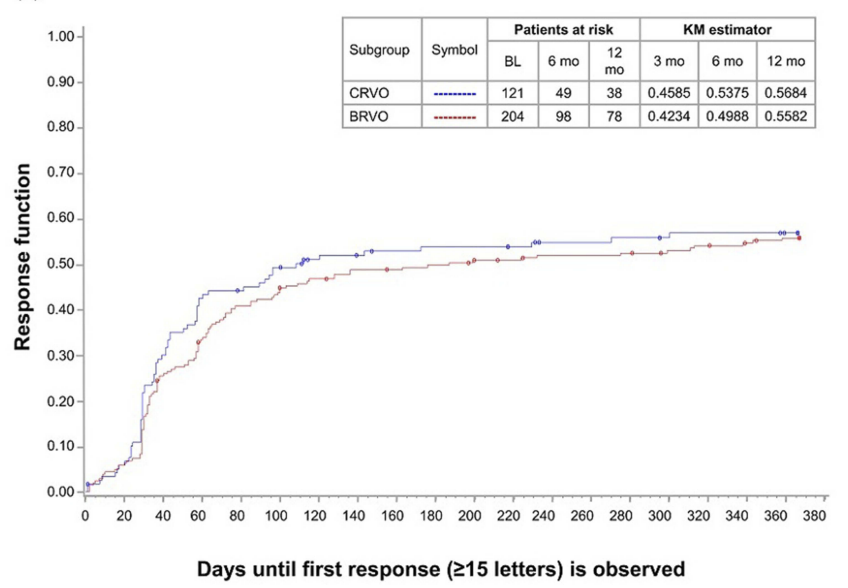

Figure 4 Time to response (BCVA improvement of $\geq 15$ ETDRS letters from baseline). (A) In all patients and (B) In BRVO and CRVO subgroups.

Notes: Time to response was defined as the first time an improvement of $\geq 15$ ETDRS letters from baseline was reached. Participants who did not reach a response were censored at the last documented time point. If no visual acuity data were documented after baseline, the participant was censored on day I. Estimators are based on the Kaplan-Meier product-limit methodology.

Abbreviations: BCVA, best-corrected visual acuity; BL, baseline; BRVO, branch retinal vein occlusion; CRVO, central retinal vein occlusion; ETDRS, Early Treatment for Diabetic Retinopathy Study; KM, Kaplan-Meier; mo, months.

(a)

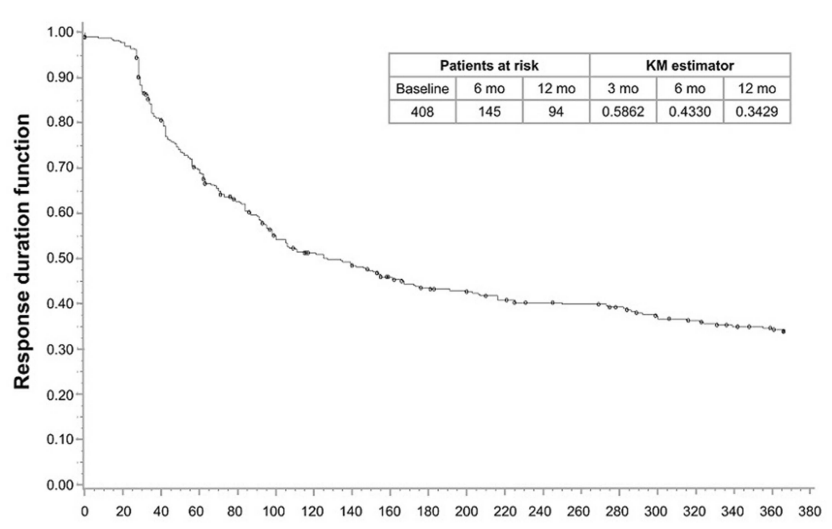

Duration of response in days after first response ( $\geq 15$ letters) was observed (b)

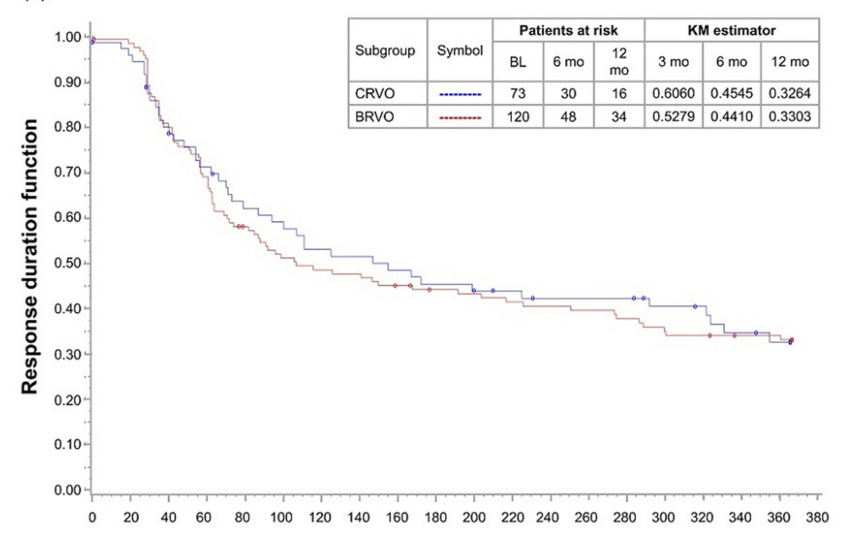

Duration of response in days after first response ( $\geq 15$ letters) was observed

Figure 5 Duration of response (time from first BCVA improvement of $\geq 15$ ETDRS letters to losing this improvement). (A) In all patients and (B) In BRVO and CRVO subgroups.

Notes: Duration of response was defined as the time from first improvement of $\geq 15$ ETDRS letters from baseline to the first time point of losing this improvement. Participants who did not lose the response were censored at the last documented time point where the response was still present. If no visual acuity data were documented after the response date, the participant was censored on day I. Estimators are based on the Kaplan-Meier product-limit methodology.

Abbreviations: BCVA, best-corrected visual acuity; BL, baseline; BRVO, branch retinal vein occlusion; CRVO, central retinal vein occlusion; ETDRS, Early Treatment for Diabetic Retinopathy Study; KM, Kaplan-Meier; mo, months.

(supporting information: Supplementary Table 1). Serious adverse events occurred in $9 \%$ of patients, with retinal vein occlusion and vitreous haemorrhage (each in 6 patients) most frequently documented. Overall, 7 patients $(0.9 \%)$ experienced glaucoma, 6 patients $(1.3 \%)$ died, and 2 patients each $(0.3 \%$ each $)$ experienced a cerebrovascular accident, underwent a vitrectomy and experienced retinal neovascularization during the study.

\section{Discussion}

In several studies, ranibizumab has been shown to be both safe and effective in treating ME associated with RVO.,7-11,14,15 However, it has also been observed that in routine clinical practice, patients with ME secondary to RVO receive fewer anti-VEGF injections than in RCTs. ${ }^{16}$ This may compromise the VA outcomes, as has been suspected in neovascular agerelated macular degeneration (nAMD). ${ }^{17}$ 
The current analysis of the RVO patient population from the non-interventional OCEAN study reports results for the 744 patients enrolled for treatment of ME secondary to RVO. These real-world data suggest that, although treatment responses were clearly seen, the numbers of BCVA and OCT examinations and ranibizumab injections over the course of 24 months were lower than recommended. Even in view of the great variability of venous occlusions - with a high chance of spontaneous improvement in mild manifestations - undertreatment was suspected. Interpretation of some data is limited by incomplete data and loss to follow-up, especially at later time points due to the non-interventional design of the study.

Patients remained in the study for a mean duration of approximately 17 months, which represents an acceptable adherence for a non-interventional study. However, a substantial number of patients were lost to follow-up. Study data are available for $74 \%$ of patients at the 12 -month visit and for $56 \%$ of patients at the 24-month visit. Loss to follow-up is to be expected in a non-interventional study, and is not necessarily the same as discontinuation of therapy. However, the reasons for discontinuation were not clearly documented for all cases in this study. It is possible that the treatment burden of a regular monitoring and injection schedule was not acceptable for at least some patients, which would support the use of T\&E regimens where the injection intervals increase over time in the absence of disease activity. Therefore, anti-VEGF drugs with increased durability would be favorable.

The presented time-to-event analyses assessed the time until study discontinuation as well as the time until treatment response and response duration. Analyses based on Kaplan-Meier estimators, especially for treatment response, are more accurate than LOCF analyses, which may imply BCVA stability over time even in patients that were lost to follow-up.

\section{Number Of Injections}

During the ranibizumab the first 3 months of the study, all 744 RVO patients received an injection at baseline and the majority also received injections at Month 1 and Month 2 . This is in line with the approved German product information for Lucentis ${ }^{\circledR}$ as well as with the ophthalmological societies. Overall, the median number of 5 injections documented for the RVO patients in OCEAN was lower than in recent PRNbased RCTs, where patients received a mean of around 7 to 11 ranibizumab injections in 12 months. ${ }^{11,12,18}$ The low number of injections seen in OCEAN may explain the less pronounced increases in VA compared to published RCTs.
On the other hand, the OCEAN results are comparable to published observational studies assessing ranibizumab therapy in routine clinical practice, in which patients received around 3 to 6 ranibizumab injections in 12 months. ${ }^{10,16,19}$ Real-world data from the UK, the USA and Germany on ranibizumab, aflibercept (Eylea ${ }^{\circledR}$, Bayer) and bevacizumab (Avastin $^{\circledR}$, Roche) for treatment of RVO showed annual injection frequencies of 3 to 7 injections, similar to the OCEAN cohort. ${ }^{20-23}$ Patients in routine clinical care tend to receive fewer injections than in the optimized setting of an $\mathrm{RCT}$, even if the RCT is performed using a PRN regimen. ${ }^{10}$ This suggests potential under-treatment. Possible reasons for non-adherence to treatment schedules on the patients' side have been investigated in several ophthalmological indications and may include time constraints, difficulties in travelling, reluctance to be injected. ${ }^{24-27}$ On the part of the physician, time pressure, an increasing number of patients, and not emphasizing the importance of regular treatment may also play a role. Additionally for this study, financial constraints could have also been a factor since the ranibizumab treatment procedure was not generally reimbursed by German health insurers before 2014.

The OCEAN patients underwent a median number of 15 BCVA examinations and 5 OCT examinations. Considering the mean duration of participation of around 17 months, these results indicate relatively regular monitoring of VA for many patients, but notably fewer OCT examinations than expected.

\section{VA Outcome}

Although the BCVA improvements over the course of the OCEAN study were notable, rapid and sustained, the positive outcomes were not as pronounced as those seen in previously published $\mathrm{RCTs}^{5-9,11,18}$ and in other observational studies, ${ }^{10,19}$ all of which reported a higher number of injections than in OCEAN. These studies had similar baseline VA to OCEAN for BRVO (OCEAN: mean 56 ETDRS letters; other studies: 53-60 letters) $)^{9,11,15,19}$ and slightly better baseline VA for CRVO (OCEAN: mean 44 ETDRS letters; other studies: 48-55 letters). ${ }^{8,10,14,18,19}$ Although the results of some published trials were superior to the OCEAN results, the magnitude of improvements in BCVA of PRN ranibizumab therapy was comparable, at least in BRVO. In CRVO, the OCEAN outcomes were more notably inferior to published results, which may be explained by the non-interventional design and by the usually worse response to treatment of CRVO, compared to BRVO, if not monitored closely. In a retrospective, 
observational trial by Wai et al, CRVO patients also showed less improvement compared to BRVO patients after 12 months of anti-VEGF treatment, ${ }^{28}$ likely due to the more severe nature of CRVO. In OCEAN, the CRVO subgroup showed notably less improvement compared to the BRVO patients, particularly during the first year of the study. The findings support the idea that CRVO patients may require closer monitoring and stricter injection regimens to achieve meaningful BCVA improvements. The data suggest that in routine clinical practice, CRVO patients in particular may be at a greater risk for undertreatment.

Furthermore, it appears plausible that in the overall OCEAN RVO population and the BRVO subgroup, the BCVA improvements could have been even higher than observed. This may be explained by the fact that the OCEAN patients only received a mean of 4.7 injections in the first 12 months of the study and around 6.0 injections during the entire 2-years. However, published realworld studies had a lower injection frequency, comparable with OCEAN. Despite the similarly low number of injections, the VA outcomes in CRVO were better in two published real-world studies than in OCEAN. ${ }^{10,19}$ The higher baseline VA in these two studies may explain the difference.

\section{Impact Of Baseline VA On Treatment Outcomes}

Relevant RCTs excluded patients based on their VA. ${ }^{8,9,11,14,15}$ As per the study's non-interventional design, OCEAN patients were enrolled and treated at the physicians' discretion. Therefore, patients with particularly high or low initial BCVA were not excluded from OCEAN. Of note, mean baseline BCVA (RVO: 52 letters, BRVO: 56 letters, CRVO: 44 letters) was slightly lower than in other published real-world studies in RVO, ${ }^{21,23}$ BRVO, ${ }^{19,28}$ and CRVO. ${ }^{10,19,28}$

In the OCEAN study, initial BCVA had a strong impact on visual outcomes after 1 and 2 years. BCVA improvements were greatest in patients with low initial BCVA $(<50$ letters), were less in patients with a baseline BCVA of 50-65 letters and showed hardly any absolute improvement in patients with a baseline BCVA of $>65$ letters. In the analysis of these data, the discontinuation rate has to be taken into account. Useful future analyses may include correlations between the patients' baseline BCVA and potential discontinuation. This is in line with previous studies that have consistently shown baseline BCVA to be a predictor for the maximum obtainable treatment outcomes. $^{11,14,15,18,28}$ It is therefore advantageous to diagnose RVO as early as possible and begin treatment soon after diagnosis. ${ }^{18,29}$ Particularly in the more severe form of CRVO, early treatment, close OCT monitoring, and prompt re-treatment seems to lead to better outcomes, compared to function-driven therapy regimens.

\section{Impact Of OCT Monitoring On VA Outcomes}

In a retrospective evaluation of anti-VEGF treatment in nAMD, Holz et al found evidence that more monitoring visits may be correlated with more successful maintenance of VA gains. ${ }^{17}$ In OCEAN, the number of monitoring OCTs received by the RVO patients had only a small impact on the VA outcomes. Patients who received more than 4 OCTs during the study tended toward better response than patients who received fewer OCTs. However, this trend was not consistently seen across all subgroup analyses. The weak link between the number of OCTs and the VA outcomes may be due to the fact that OCTs are not always performed when required, due to physicians' or patients' time constraints and due to the fact that OCTs are usually not reimbursed. Furthermore, the evaluation of OCT images needs experience and time to fully analyze potential re-treatment criteria.

\section{Implementation Of Clinical Trial Findings In Real World Practice}

When interpreting the OCEAN results, specific circumstances of the trial should be considered. The injection procedure was not reimbursed by the German statutory health insurance for most of the OCEAN study period; only starting in October 2014. Furthermore, OCT examinations are still not generally reimbursed by statutory insurance, depending on particular insurance contracts. This situation may limit patients' access to monitoring and timely treatment. Adherence to planned treatment schedules could be optimized by physicians sending out reminders to patients, encouraging monitoring visits and allowing early re-treatment where necessary.

\section{Limitations}

The limitations of the OCEAN study are mainly due to its non-interventional nature. By design, patients were not randomized, no control group was included, and treatment 
was not blinded. Treatments and examinations were performed at the physician's discretion. Documentation was not available for all patients at all study time points and documentation was not always complete. It must be assumed that adverse event reporting was also not complete. The study attrition rate was notably higher than in RCTs, as expected. However, time-to-event analyses demonstrated that study discontinuation was constant throughout the study. An additional limitation was the fact that for a number of patients the subgroup (BRVO or CRVO) was not specifically documented. Considering the two indications' different characteristics, ${ }^{19,30}$ it would have been beneficial to make the distinction between BRVO and CRVO for all patients.

On the other hand, the study's strengths lie in the high number of patients recruited, the broad inclusion criteria without exclusion of patients based on BCVA, and the high number of participating sites. Furthermore, the study obtained long-term results over 2 years. These factors allowed the evaluation of a broad spectrum of patients, which should provide relatively accurate and representative insight into routine clinical practice in Germany.

\section{Conclusions}

The OCEAN trial showed the beneficial effects of ranibizumab in patients with ME secondary to RVO, particularly for patients with BRVO. In patients with CRVO, the data showed less and later improvements. In the real-world setting of the OCEAN study, patients received fewer injections and fewer OCT examinations than previously reported in clinical trials. In agreement with other studies, the results demonstrate that baseline BCVA has an impact on BCVA improvement. This emphasizes the importance of an early treatment and regular follow-up to achieve good results. However, interpretation is limited due to study discontinuations and incomplete data, particularly at later time points. Future studies should address this and characterize the patient populations that discontinue treatment, including in-depth analyses of reasons for discontinuation. Safety data were consistent with the well-established profile of ranibizumab in the treatment of patients with RVO, with no new safety issues.

\section{Data Sharing Statement}

The authors have full control of all primary data. Individual participant data that underlie the results reported in this article, after deidentification will be available data upon request. Study protocol, statistical analysis plan, informed consent form will be available. Proposals may be submitted to the corresponding author up to 36 months following article publication.

\section{Acknowledgements}

The non-interventional OCEAN study was sponsored by Novartis Pharma GmbH (Nuremberg, Germany). Medical writing assistance for the preparation of this manuscript was also funded by Novartis Pharma GmbH. The authors would like to thank the project team at Kantar Health GmbH (Munich, Germany), especially Klaus Laschke for the assistance in data analysis, Daniela Müller for writing and editing assistance, and Susanne Faber for project management.

\section{Disclosure}

Josep Callizo received personal fees and research grants from Novartis and personal fees from Bayer Healthcare and Allergan. Focke Ziemssen received grants and personal fees from Biogen, Allergan, Alimera, Bayer Healthcare and Roche, grants from Clearside, personal fees from Boehringer Ingelheim, Novartis, NovoNordisk, MSD Sharp \& Dohme. Thomas Bertelmann received research grants from Novartis, personal fees from Alcon, Alimera, Allergan, Bayer, Heidelberg, Novartis, and was a Medical Advisor for Novartis (2015-2016). Nicolas Feltgen has received funds from Novartis, Allergan, Bayer, Alimera, Heidelberg Engineering, and Roche. Jessica Voegeler and Mirja Koch are Novartis employees. Nicole Eter received research grants from Novartis and Bayer, speaker's honorarium from Novartis, Bayer, Allergan, Heidelberg Engineering, and Alimera, and served on Advisory Boards for Novartis, Bayer, Allergan, Alimera, and Roche. Sandra Liakopoulos received speaker's fees from Novartis, Carl Zeiss Meditec, Heidelberg Engineering, Allergan, Bayer; non-financial research support from Carl Zeiss Meditec and Heidelberg Engineering and was member of an advisory board for Novartis. Steffen Schmitz-Valckenberg received grants and non-financial research funding from Accucela, Alcon/ Novartis, Allergan, Bayer, Bioeq/Formycon, Centervue, Genentech/Roche, Heidelberg Engineering, Optos, Carl Zeiss MediTec, and personal fees and honoraria from Alcon/Novartis, Bayer, Genentech/Roche, Carl Zeiss MediTec. Georg Spital received personal fees from Bayer Healthcare, Novartis, Carl Zeiss Meditec, OD-OS. The authors report no other conflicts of interest in this work. 


\section{References}

1. Ho M, Liu DT, Lam DS, Jonas JB. Retinal vein occlusions, from basics to the latest treatment. Retina. 2016;36(3):432-448. doi:10.1097/IAE.0000000000000843

2. Yau JW, Lee P, Wong TY, Best J, Jenkins A. Retinal vein occlusion: an approach to diagnosis, systemic risk factors and management. Intern Med J. 2008;38(12):904-910. doi:10.1111/j.1445-5994.2008.01720.x

3. Braithwaite T, Nanji AA, Lindsley K, Greenberg PB. Anti-vascular endothelial growth factor for macular oedema secondary to central retinal vein occlusion. Cochrane Database Syst Rev. 2014;(5):CD007325.

4. McIntosh RL, Rogers SL, Lim L, et al. Natural history of central retinal vein occlusion: an evidence-based systematic review. Ophthalmology. 2010;117(6):1113-1123 e1115. doi:10.1016/j.ophtha.2010.01.060

5. Brown DM, Campochiaro PA, Bhisitkul RB, et al. Sustained benefits from ranibizumab for macular edema following branch retinal vein occlusion: 12-month outcomes of a phase III study. Ophthalmology. 2011;118(8):1594-1602. doi:10.1016/j.ophtha.2011.02.022

6. Campochiaro PA, Brown DM, Awh CC, et al. Sustained benefits from ranibizumab for macular edema following central retinal vein occlusion: twelve-month outcomes of a phase III study. Ophthalmology. 2011;118(10):2041-2049. doi:10.1016/j.ophtha.2011.02.038

7. Heier JS, Campochiaro PA, Yau L, et al. Ranibizumab for macular edema due to retinal vein occlusions: long-term follow-up in the HORIZON trial. Ophthalmology. 2012;119(4):802-809. doi:10.1016/j. ophtha.2011.12.005

8. Hoerauf H, Feltgen N, Weiss C, et al. Clinical efficacy and safety of ranibizumab versus dexamethasone for Central Retinal Vein Occlusion (COMRADE C): a European label study. $\mathrm{Am} J$ Ophthalmol. 2016;169:258-267. doi:10.1016/j.ajo.2016.04.020

9. Hattenbach LO, Feltgen N, Bertelmann T, et al. Head-to-head comparison of ranibizumab $\mathrm{PRN}$ versus single-dose dexamethasone for branch retinal vein occlusion (COMRADE-B). Acta Ophthalmol (Copenh). 2018;96(1):e10-e18. doi:10.1111/aos.13381

10. Chatziralli I, Theodossiadis G, Kabanarou SA, et al. Ranibizumab versus dexamethasone implant for central retinal vein occlusion: the RANIDEX study. Graefe's Archive Clin Exp Ophthalmol. 2017;255 (10):1899-1905. doi:10.1007/s00417-017-3719-5

11. Tadayoni R, Waldstein SM, Boscia F, et al. Sustained benefits of ranibizumab with or without laser in branch retinal vein occlusion: 24-month results of the BRIGHTER study. Ophthalmology. 2017;124 (12):1778-1787. doi:10.1016/j.ophtha.2017.06.027

12. Feltgen N, Hattenbach LO, Bertelmann T, et al. Comparison of ranibizumab versus dexamethasone for macular oedema following retinal vein occlusion: 1-year results of the COMRADE extension study. Acta Ophthalmol (Copenh). 2018. doi:10.1111/aos.13770

13. Ziemssen F, Feltgen N, Holz FG, et al. Demographics of patients receiving intravitreal anti-VEGF treatment in real-world practice: healthcare research data versus randomized controlled trials. $B M C$ Ophthalmol. 2017;17(1):7. doi:10.1186/s12886-017-0401-y

14. Brown DM, Campochiaro PA, Singh RP, et al. Ranibizumab for macular edema following central retinal vein occlusion: six-month primary end point results of a phase III study. Ophthalmology. 2010;117(6):1124-1133 e1121. doi:10.1016/j.ophtha.2010.02.022

15. Campochiaro PA, Heier JS, Feiner L, et al. Ranibizumab for macular edema following branch retinal vein occlusion: six-month primary end point results of a phase III study. Ophthalmology. 2010;117 (6):1102-1112 e1101. doi:10.1016/j.ophtha.2010.02.021

16. Kiss S, Liu Y, Brown J, et al. Clinical utilization of anti-vascular endothelial growth-factor agents and patient monitoring in retinal vein occlusion and diabetic macular edema. Clin Ophthalmol. 2014;8:1611-1621. doi:10.2147/OPTH.S60893
17. Holz FG, Tadayoni R, Beatty S, et al. Multi-country real-life experience of anti-vascular endothelial growth factor therapy for wet agerelated macular degeneration. Br J Ophthalmol. 2015;99(2):220-226. doi:10.1136/bjophthalmol-2014-305327

18. Larsen M, Waldstein SM, Boscia F, et al. Individualized ranibizumab regimen driven by stabilization criteria for central retinal vein occlusion: twelve-month results of the CRYSTAL study. Ophthalmology. 2016;123(5):1101-1111. doi:10.1016/j.ophtha.2016.01.011

19. Khan M, Wai KM, Silva FQ, et al. Comparison of ranibizumab and bevacizumab for macular edema secondary to retinal vein occlusions in routine clinical practice. Ophthalmic Surg Lasers Imaging Retina. 2017;48(6):465-472. doi:10.3928/2325816020170601-04

20. Lip PL, Malick H, Damer K, et al. One-year outcome of bevacizumab therapy for chronic macular edema in central and branch retinal vein occlusions in real-world clinical practice in the UK. Clin Ophthalmol. 2015;9:1779-1784. doi:10.2147/OPTH.S89147

21. Jumper JM, Dugel PU, Chen S, Blinder KJ, Walt JG. Anti-VEGF treatment of macular edema associated with retinal vein occlusion: patterns of use and effectiveness in clinical practice (ECHO study report 2). Clin Ophthalmol. 2018;12:621-629. doi:10.2147/OPTH.S163859

22. Lotery AJ, Regnier S. Patterns of ranibizumab and aflibercept treatment of central retinal vein occlusion in routine clinical practice in the USA. Eye (Lond). 2015;29(3):380-387. doi:10.1038/eye.2014.308

23. Wecker T, Ehlken C, Buhler A, et al. Five-year visual acuity outcomes and injection patterns in patients with pro-re-nata treatments for AMD, DME, RVO and myopic CNV. Br J Ophthalmol. 2017;101 (3):353-359. doi:10.1136/bjophthalmol-2016-308668

24. Ehlken C, Helms M, Boehringer D, Agostini HT, Stahl A. Association of treatment adherence with real-life VA outcomes in AMD, DME, and BRVO patients. Clin Ophthalmol. 2018;12:13. doi:10.2147/OPTH.S151611

25. Framme C, Eter N, Hamacher T, et al. Aflibercept for patients with neovascular age-related macular degeneration in routine clinical practice in Germany. Ophthalmol Retina. 2018;2(6):539-549. doi:10.1016/ j.oret.2017.09.017

26. Boulanger-Scemama E, Querques G, About F, et al. Ranibizumab for exudative age-related macular degeneration: a five year study of adherence to follow-up in a real-life setting. J Fr Ophtalmol. 2015;38(7):620-627. doi:10.1016/j.jfo.2014.11.015

27. Droege KM, Muether PS, Hermann MM, et al. Adherence to ranibizumab treatment for neovascular age-related macular degeneration in real life. Graefe's Archive Clin Exp Ophthalmol. 2013;251(5):12811284. doi:10.1007/s00417-012-2177-3

28. Wai KM, Khan M, Srivastava S, et al. Impact of initial visual acuity on anti-VEGF treatment outcomes in patients with macular oedema secondary to retinal vein occlusions in routine clinical practice. Br J Ophthalmol. 2017;101(5):574-579. doi:10.1136/ bjophthalmol-2016-308727

29. Ziemssen F, Bertelmann T, Hufenbach U, Scheffler M, Liakopoulos S, Schmitz-Valckenberg S. Verzoegerung des Behandlungsbeginns um mehr als 2 Wochen. Relevanz fur moeglichen Visusgewinn nach antiVEGF-therapie unter real-life-bedingungen (Interimanalyse der prospektiven OCEAN-studie). Der Ophthalmologe. 2016;113(2):143-151. doi:10.1007/s00347-015-0099-2

30. Koss MJ, Pfister M, Rothweiler F, et al. Comparison of cytokine levels from undiluted vitreous of untreated patients with retinal vein occlusion. Acta Ophthalmol (Copenh). 2012;90(2):e98-e103. doi:10.1111/j.1755-3768.2011.02292.x 


\section{Publish your work in this journal}

Clinical Ophthalmology is an international, peer-reviewed journal covering all subspecialties within ophthalmology. Key topics include: Optometry; Visual science; Pharmacology and drug therapy in eye diseases; Basic Sciences; Primary and Secondary eye care; Patient Safety and Quality of Care Improvements. This journal is indexed on PubMed

Central and CAS, and is the official journal of The Society of Clinical Ophthalmology (SCO). The manuscript management system is completely online and includes a very quick and fair peer-review system, which is all easy to use. Visit http://www.dovepress.com/ testimonials.php to read real quotes from published authors. 